\title{
Adrenergic modulation of AMPK-dependent autophagy by chronic stress enhances cell proliferation and survival in gastric cancer
}

\author{
XIAOFEI ZHI ${ }^{1,2^{*}}$, BOWEN LI ${ }^{1 *}$, ZHENG LI ${ }^{1}$, JIAXUAN ZHANG ${ }^{3}$, \\ $\mathrm{JUNBO} \mathrm{YU}^{3}$, LU ZHANG ${ }^{1}$ and ZEKUAN XU ${ }^{1}$ \\ ${ }^{1}$ Department of General Surgery, The First Affiliated Hospital of Nanjing Medical University, \\ Nanjing, Jiangsu 210029; Departments of ${ }^{2}$ Gastrointestinal Surgery, and ${ }^{3}$ Emergency Surgery, \\ Affiliated Hospital of Nantong University, Nantong, Jiangsu 226001, P.R. China
}

Received September 10, 2018; Accepted February 22, 2019

DOI: 10.3892/ijo.2019.4753

\begin{abstract}
Epidemiological data show that chronic stress has adverse effects on the incidence and progression of cancer. As a critical target organ for stress hormones, the stomach is frequently subjected to stress-related injury. However, few reports regarding the association between stress and gastric cancer (GC) have been published. The present study aimed to investigate the effect of chronic stress on the growth and survival of GC, and the role of the autophagy process. A restraint-stress procedure over 21 days was used to establish a chronic stress mouse model. Subcutaneous xenografts and gastric orthotopic xenografts were established in BALB/c nude mice. Alzet osmotic minipumps containing either PBS or propranolol hydrochloride was inserted on the nape of the neck 7 days prior to the initiation of restraint stress. The presence of autophagosomes and autolysosomes were examined by electron microscopy. The stress hormone norepinephrine significantly enhanced the proliferation of GC cells. By inhibiting adrenoreceptor expression, it was demonstrated that $\beta 2$-adrenergic receptor (ADRB2) was the specific $\beta$-adrenergic
\end{abstract}

Correspondence to: Mr. Zekuan Xu, Department of General Surgery, The First Affiliated Hospital of Nanjing Medical University, 300 Guangzhou Road, Nanjing, Jiangsu 210029, P.R. China

E-mail: xuzekuan@njmu.edu.cn

\section{*Contributed equally}

Abbreviations: GC, gastric cancer; ADRB2, $\beta 2$-adrenergic receptor; DFS, disease-free survival; TMA, tissue microarray; IRS, immunoreactivity score; PVDF, polyvinylidene fluoride; ADRB1, $\beta 1$-adrenergic receptor; NC, negative control; TEM, transmission electron microscopy; TUNEL, terminal-deoxynucleoitidyl transferase mediated nick end labeling; AMPK, adenosine 5'-monophosphate-activated protein kinase; CREB, cAMP-response element binding protein; PKA, protein kinase $\mathrm{A}$

Key words: chronic stress, gastric cancer, autophagy receptor subtype responsible for catecholamine release. In addition, it was demonstrated that the induction of autophagy was a novel consequence of $\beta 2$-adrenergic activation in GC cells. This was demonstrated by the appearance of double-membrane vesicles, punctuate GFP-RFP-microtubule-associated protein 1 light chain 3 distribution in the cytoplasm and a corresponding increase in autophagic flux. Notably, norepinephrine-induced autophagy was shown to have a tumor-promoting role under conditions of chronic stress in vitro and in vivo. It was further demonstrated that, upon activation of cAMP-response element binding protein, chronic stress promoted autophagic flux through the adenosine 5'-monophosphate-activated protein kinase-unc-51 like autophagy activating kinase 1 (AMPK-ULK1) pathway. Tissue microarray analysis revealed a negative correlation between the expression of ADRB2 and autophagic marker p62/sequestosome-1 in GC tumor samples. Additionally, high protein levels of ADRB2 correlated positively with tumor, node, metastasis stage and poor prognosis in patients with GC. These results establish a novel pathway that chronic stress activates tumor-promoting autophagy to accelerate the progression of GC. The present study is the first, to the best of our knowledge, providing preclinical evidence that chronic stress serves a role in the progression of GC.

\section{Introduction}

Epidemiological data show that chronic stress in a negative social and psychological state has an adverse effect on cancer incidence and progression (1-3). Additionally, patients with cancer have increased stress levels, which further exacerbates cancer progression (4). Laboratorial studies have demonstrated that catecholamines released from the hypothalamic-pituitary-adrenal axis in response to stressors not only affect cellular immunity, but also contribute to tumor proliferation, metastasis and angiogenesis through various signaling pathways (5-9). As an important target organ of stress hormones, the stomach is frequently subjected to stress-related injury, such as stress ulcers (10). However, few reports on the association between stress and gastric cancer (GC) have been published to date. 
Autophagy is an evolutionarily conserved process in eukaryotes. It is a process that degrades cytoplasmic components, including long-lived, damaged proteins, and organelles (11). The by-products of autophagy are recycled back to the cytosol as macromolecules for the synthesis of new structures (12). The process of autophagy can be divided into three stages: Sequestration of cytosolic components by the autophagosome, fusion of autophagosomes with lysosomes to form autolysosomes and degradation of the components incorporated into autolysosomes. Dysregulation in autophagy has been associated with several human disorders, including metabolic diseases and cancer (13). Under different stress conditions, autophagy primarily represents an essential adaptive response to provide nutrients and energy (14). Increasing evidence has demonstrated that autophagy is a key component of the stress response in cancer cells (15-17). It is generally considered that autophagy has two primary and opposing functions in tumor cells in response to stress, the cytoprotective function and the cytotoxic function (11). Autophagy can act as a tumor suppressor by eliminating oncogenic proteins and preventing oxidative stress and genome instability. By contrast, autophagy can promote tumor development by providing substrates that allow tumor cells to overcome nutrient limitation and hypoxia (18). However, the effects of stress hormones on GC cells and the role of stress hormone-induced autophagy remain to be elucidated.

The present study is the first, to the best of our knowledge, to provide direct preclinical evidence for the role of chronic stress in the progression of GC. In addition, the present study identified the positive role of autophagy on the norepinephrine-induced progression of GC and revealed an important regulatory mechanism of the activation of ADRB2 signaling in autophagy.

\section{Materials and methods}

Cell lines and cell culture. The SGC-7901 and BGC-823 human GC cell lines were purchased from the Shanghai Institutes for Biological Sciences, Chinese Academy of Sciences (Shanghai China), and were incubated in RPMI-1640 (Gibco; Thermo Fisher Scientific, Inc., Waltham, MA, USA) supplemented with 10\% FBS (Gibco; Thermo Fisher Scientific, Inc.). All cell lines were grown in a humidified chamber supplemented with $5 \% \mathrm{CO}_{2}$ at $37^{\circ} \mathrm{C}$. Norepinephrine (10 $\mu \mathrm{M}$; Sigma, Shanghai, China), $5 \mu \mathrm{M}$ terbutaline (Sigma), $1 \mu \mathrm{M}$ xamoterol (Tocris Chemicals, Shanghai, China), $10 \mu \mathrm{M}$ propranolol (Sigma), $50 \mathrm{nMH} 89$ 2HCL (Selleck Chemicals, Shanghai, China), $5 \mu \mathrm{M}$ Chloroquine (Selleck Chemicals) in PBS were used to treat the cells for $24 \mathrm{~h}$ prior to testing. In addition, $5 \mu \mathrm{g} / \mathrm{ml} \mathrm{E64d} \mathrm{(Sigma)} \mathrm{and} 5 \mu \mathrm{g} / \mathrm{ml}$ pepstain A (Sigma) were used to treat the cells following norepinephrine treatment.

Patients and tissue microarray (TMA). Paired tumor and adjacent non-tumor human gastric tissues were obtained from 133 patients with GC who underwent radical resection at the First Affiliated Hospital of Nanjing Medical University (Nanjing, China) between 2008 and 2010. All patients were diagnosed pathologically according to the criteria of the American Joint Committee on Cancer (19) by two professional pathologists independently. The clinicopathological details are provided in Table I. Disease-free survival (DFS) was the primary end-point. Formalin-fixed, paraffin-embedded tissues were used to construct a TMA, as described previously (20). The thickness of the tissue section was $3 \mu \mathrm{m}$. All procedures performed in experiments involving human participants were in accordance with the ethical standards of the institutional and/or national research committee and with the 1964 Helsinki declaration and its later amendments or comparable ethical standards. The present study was approved by the Institutional Ethical Board of the First Affiliated Hospital of Nanjing Medical University. Informed consent was obtained from all individual participants included in the study.

Immunohistochemistry and assessment. The tissue slide was heated at $60^{\circ} \mathrm{C}$ for $1 \mathrm{~h}$, followed by treatment with xylene, $100 \%$ ethanol and then decreasing concentrations of ethanol. Following antigen retrieval, the tissues were blocked with $5 \%$ bovine serum albumin (BSA) (Roche, Basel, Switzerland) for $30 \mathrm{~min}$ at room temperature, and stained with antibodies at $4^{\circ} \mathrm{C}$ overnight, followed by incubation with biotinylated secondary antibody (1:200) for $30 \mathrm{~min}$ at $37^{\circ} \mathrm{C}$ and visualizing using a standard avidin biotinylated peroxidase complex method. The following antibodies were used: ADRB2 (cat. no. ab61778, 1:100, Abcam, Cambridge, UK), SQSTM1 (cat. no. ab56416, 1:100, Abcam), Ki-67 (cat. no. ab15580, 1:100, Abcam) and biotinylated goat anti-rabbit IgG (cat. no. ab64256, 1:200, Abcam). Immunostaining was observed under a Zeiss fluorescence microscope at a x100 or x200 magnification.The immunoreactivity was scored independently by two pathologists using a semi-quantitative immunoreactivity score (IRS) (21). The IRS was calculated by combining the quantity score with the intensity score. Briefly, the quantity score represented the percentage of immunoreactive cells as $1(0-25 \%), 2$ (26-50\%), 3 (51-75\%) and $4(76-100 \%)$. The intensity score represented the intensity of immunostaining as 0 (negative), 1 (weak), 2 (moderate) and 3 (strong). Multiplication of the quantity score and intensity score resulted in an IRS ranging between 0 and 12 . The immunoreactivity was assessed as follows: - (score 0); + (score $1-4) ;++($ score $5-8) ;+++$ (score $9-12)$. IRS $\leq 4$ was divided into a low expression group and IRS $>4$ was divided into a high expression group.

Reverse transcription-quantitative polymerase chain reaction (RT-qPCR) analysis. Total RNA was extracted from the paired tumorous and adjacent non-tumorous human gastric tissues with TRIzol reagent (Invitrogen; Thermo Fisher Scientific, Inc.) according to the manufacturer's protocol, and was reverse-transcribed into cDNA with PrimeScript RT reagent (Takara Biotechnology Co., Ltd., Dalian, China). qPCR was performed using a 7500 Real-time PCR system (Applied Biosystems; Thermo Fisher Scientific, Inc.) with a SYBR Premix Ex Taq kit (Takara Biotechnology Co., Ltd.). The following primers were used: ADRB2, forward 5'-AGAGCCT GCTGACCAAGAAT-3' and reverse 5'-TAGCAGTTGATGGC TTCCTG-3'; $\beta$-actin, forward 5'-AGAGCCTCGCCTTTGCCG ATCC-3' and reverse 5'-CTGGGCCTCGTCGCCCACATA-3'. The cycling conditions were comprised of $95^{\circ} \mathrm{C}$ for $10 \mathrm{~min}$, followed by 40 cycles of $95^{\circ} \mathrm{C}$ for $15 \mathrm{sec}$ and $60^{\circ} \mathrm{C}$ for $1 \mathrm{~min}$. 
Table I. Clinical characteristics and expression of ADRB2 in 133 patients with gastric cancer.

\begin{tabular}{lcccc}
\hline & \multicolumn{4}{c}{ ADRB2 expression } \\
\cline { 4 - 5 } Clinical & No. of patients & High & Low & P-value \\
characteristic & $(\mathrm{n}=133)$ & $(\mathrm{n}=53)$ & $(\mathrm{n}=80)$ & \\
\hline Age (years $)$ & 63 & 30 & 33 & 0.110 \\
$\leq 60$ & 70 & 23 & 47 & \\
$>60$ & & & & \\
Sex & 81 & 33 & 48 & 0.857 \\
Male & 52 & 20 & 32 & \\
Female & & & & \\
Differentiation & 62 & 20 & 42 & 0.112 \\
Well/moderate & 71 & 33 & 38 & \\
Poor & & & & \\
TNM stage & 21 & 3 & 18 & 0.014 \\
Stage I & 112 & 50 & 62 & \\
Stage II/III & & & & \\
\hline
\end{tabular}

ADRB2, $\beta 2$-adrenergic receptor; TNM, tumor-node-metastasis.

The $2^{-\Delta \Delta C q}$ method was used to calculate relative expression (22). All procedures were performed in triplicate.

Western blotting. Total proteins were extracted using lysis buffer [50 mM Tris-HCl ( $\mathrm{pH} 7.4), 150 \mathrm{mM} \mathrm{NaCl}$, $1 \%$ Triton $\mathrm{X}-100,0.1 \%$ SDS, $1 \mathrm{mM}$ EDTA and protease inhibitor cocktail]. The concentrations were determined using the Bradford assay. Proteins (30 $\mu \mathrm{g} /$ lane) were loaded on concentration-appropriate gels. The cellular protein was size-fractionated by $10 \%$ SDS-polyacrylamide gel electrophoresis and transferred onto PVDF membranes (Bio-Rad Laboratories, Inc., Hercules, CA, USA). Following blocking with PBS containing 5\% BSA for $2 \mathrm{~h}$ at room temperature, the membrane was incubated with the appropriate primary antibodies at $4^{\circ} \mathrm{C}$ overnight, followed by incubation with HRP-conjugated anti-mouse or anti-rabbit IgG (cat. no. ab6728 and cat. no. ab6721, 1:1,000, Abcam) at room temperature for $2 \mathrm{~h}$. The protein bands were detected using an enhanced chemiluminescence detection system following the manufacturer's protocol. Anti-microtubule-associated protein 1 light chain 3 (LC3) A/B (cat. no. 4108, 1:1,000), anti-p62 (cat. no. 88588, 1:1,000), anti-adenosine 5'-monophosphate (AMP)-activated protein kinase (AMPK) (cat. no. 5832, 1:1,000), anti-phosphorylated (p-)AMPK (Thr172) (cat. no. 50081, 1:1,000), anti-unc-51 like autophagy activating kinase 1 (ULK1) (cat. no. 6439, 1:1,000), anti-p-ULK1 (Ser555) (cat. no. 5869, 1:1,000), anti-p-CREB (Ser133) (cat. no. 9198, 1:1,000) and anti-CREB (cat. no. 9197, 1:1,000) were obtained from Cell Signaling Technology, Inc. (Danvers, MA, USA). Anti-ADRB2 (cat. no. ab61778, 1:1,000), anti-Beclin 1 (cat. no. ab62557, 1:1,000), anti-caspase-3 (cat. no. ab13847, 1:1,000), anti- $\beta$-catin (cat. no. ab8227, 1:1,000) antibodies were obtained from Abcam. All procedures were performed in triplicate. Protein expression was semi-quantified using ImageJ version 1.46 software (National Institutes of Health, Bethesda, MD, USA).

Cell viability assay. The cells (2,000/well) were seeded into 96-well plates and stained at the indicated time point using the Cell Counting Kit-8 (Dojindo Molecular Laboratories, Inc., Kumamoto, Japan), according to the manufacturer's protocol. The optical density measured at $450 \mathrm{~nm}$ was used as an indicator of cell viability. All procedures were performed in triplicate.

Colony formation assay. The GC cells were cultured in 6-well plates (500 cells/well). Following treatment, the cells were cultured for a further 3 weeks. Colonies composed of $\geq 50$ or more cells were identified as positive colonies. The cells were stained with $0.2 \%$ crystal violet (KeyGEN, Jiangsu, China) for $20 \mathrm{~min}$ at room temperature. Colonies were observed under a Zeiss microscope at a x100 magnification. All procedures were performed in triplicate.

Cell apoptosis analysis. Flow cytometry and terminal-deoxynucleoitidyl transferase-mediated nick end labeling (TUNEL) were used to analyze cell apoptosis. Flow cytometry was used to analyze cell apoptosis. The cells were treated with norepinephrine for $24 \mathrm{~h}$ prior to being harvested. The staining of apoptotic cells was achieved by incubating the cells with7-AAD and Annexin V-Alexa Fluor 647 (BD Biosciences, Franklin Lakes, NJ, USA) in the dark for $15 \mathrm{~min}$ at room temperature. The cells were then examined on a FACScan flow cytometer (BD Biosciences). All procedures were performed in triplicate. TUNEL assays were conducted using the TUNEL apoptotic cell detection kit (Roche), according to the manufacturer's instructions.

Electron microscopy. The cells were pre-treated with $10 \mu \mathrm{M}$ norepinephrine for $24 \mathrm{~h}$. For electron microscopy, the cells were trypsinized using $0.25 \%$ trypsin (Thermo Fisher Scientific, Shanghai, China) for $1 \mathrm{~min}$ at $37^{\circ} \mathrm{C}$ and frozen at high pressure. The frozen samples were rapidly transferred to a Leica EM AFS2 freeze subsystem at $-90^{\circ} \mathrm{C}$ for $96 \mathrm{~h}$. The temperature was then gradually increased to $-60^{\circ} \mathrm{C}$ for $28 \mathrm{~h}$ and then to $-20^{\circ} \mathrm{C}$. The samples were dehydrated with absolute ethanol and were infiltrated with LR gold resin. The ultrathin sections $(50-70 \mathrm{~nm})$ were stained with $4 \%$ uranyl acetate and lead citrate. Images were captured using a Technai G2 Spirit TWIN transmission electron microscope (FEI; Thermo Fisher Scientific, Inc.) equipped with a Gatan 1 k x 1 k CCD camera. Quantification was performed by counting individual autophagosomes and autolysosomes in 24 random cell sections of $100-\mu \mathrm{m}^{2}$ cytoplasmic area.

Immunofluorescence. The cells were pre-treated with $10 \mu \mathrm{M}$ norepinephrine for $24 \mathrm{~h}$. The cells were then fixed with 4\% paraformaldehyde for $15 \mathrm{~min}$, permeabilized and blocked with blocking buffer (KeyGEN) containing 1\% BSA, $0.3 \mathrm{M}$ glycine, $0.1 \%$ Tween-20 and 5\% donkey serum in PBS (pH 8.0) for $1 \mathrm{~h}$ at room temperature. Following incubation with antibodies (LC3A, cat. no. 4599, 1:200 and LC3B, cat. no. 2775, 1:200; Cell Signaling Technology) overnight at $4^{\circ} \mathrm{C}$, the cells were washed with PBS and labeled with fluorescence-conjugated secondary antibodies (cat. no. 4412, 1:1,000, and cat. no. 8889, 
1:1,000;Cell Signaling Technology) for $2 \mathrm{~h}$ at room temperature and counterstained with DAPI. Images were captured using a Zeiss LSM 780 confocal laser scanning microscope.

GFP-mRFP-LC3 imaging. A lentivirus carrying GFP-mRFP-LC3 was purchased from Shanghai GeneChem Co., Ltd. (Shanghai, China) and used to infect GC cells following the manufacturer's protocol. The transfected cells underwent puromycin selection for 2 months. The cells were determined by confocal microscopy (Carl Zeiss, Oberkochen, Germany) following Hoechst $33342(2 \mu \mathrm{g} / \mathrm{ml})$ staining for $10 \mathrm{~min}$ at room temperature. Red puncta represent autolysosomes and yellow' puncta represent autophagosomes. The numbers of puncta were calculated per cell in five high-power fields.

Animal models. Male BALB/c nude mice (5weeks old, weighing $\sim 20 \mathrm{~g}$ ) were purchased from Vital River Laboratories, Co., Ltd. (Nanjing, China) and were housed in the Laboratory Animal Centre of Nanjing Medical University under the following conditions: Temperature, $22-25^{\circ} \mathrm{C}$; humidity, 50-60\%; $12 \mathrm{~h} \mathrm{light/dark} \mathrm{cycle.} \mathrm{Mice} \mathrm{had} \mathrm{free} \mathrm{access} \mathrm{to} \mathrm{water} \mathrm{and} \mathrm{food.}$ Mice were randomly divided into 6 groups (6 mice per group). The GC cells $\left(10^{6}\right.$ cells $/ 100 \mu 1$ per mouse $)$ were inoculated subcutaneously to form the first-generation xenografts. The xenografts were serially transplanted into the gastric wall by inoculating tumor fragments $(2 \times 2 \times 2 \mathrm{~mm})$ through laparotomy. The kinetics of tumor formation were assessed by measuring the tumor sizes every 4 days. The tumor volume was calculated using the following formula: Volume $=\left(\right.$ width $^{2} \mathrm{x}$ length $) / 2$. For the chronic stress model, a restraint-stress procedure was used for 21 days based on a previous study (9). For $\beta$-blockade, Alzet osmotic minipumps (Durect Corporation, Cupertino, CA, USA) containing PBS or $2 \mathrm{mg} / \mathrm{kg} / \mathrm{d}$ propranolol hydrochloride (Sigma-Aldrich; Merck KGaA, Darmstadt, Germany) were inserted on the nape of the neck 7 days prior to the initiation of restraint stress (9). The experimental protocols were approved by the Animal Care and Use Committee of the Laboratory Animal Center of Nantong University.

Plasma sampling and catecholamine assay. The concentrations of stress hormones (norepinephrine and corticosterone) were determined in plasma samples collected immediately following the removal of the animals from the stress situation. The mice were anesthetized and $\sim 500 \mu \mathrm{l}$ blood was withdrawn by cardiac puncture using a 1-ml plastic syringe. Blood was centrifuged at $1,100 \mathrm{~g}$ for $10 \mathrm{~min}$ at $4^{\circ} \mathrm{C}$. Plasma was collected and stored at $-80^{\circ} \mathrm{C}$ until required. The catecholamine concentration in plasma samples was measured by high performance liquid chromatography with electrochemical detection, as previously described (23).

RNA interference and plasmids. The synthesized DNA fragments encoding the short hairpin RNA (shRNA) used for the knockdown of endogenous ADRB2 were inserted into the pGPU6/GFP/Neo vector (GenePharma, Co. Ltd., Shanghai, China). The sequences of the shRNAs were as follows: ADRB1-shRNA, 5'-CCGATAGCAGGTGAACTCGAACTC GAGTTCGAGTTCACCTGCTATCGG-3', ADRB2-shRNA, 5'-GCATCGTCATGTCTCTCATCGCTCGAGCGATGAGA GACATGACGATGC-3', negative control (NC)-shRNA, 5'-TTC
ACCGAACGTGAAACGTATCGAGACGTGACAAGTTCG GAGAA-3'. All plasmids were verified by sequencing.

Statistical analysis. Statistical analysis was performed with SPSS software (SPSS Standard version 13.0; SPSS, Chicago, IL, USA). Data were analyzed using two-tailed Student's t-tests for two groups. Datasets containing more than two groups were compared using one-way analysis of variance and Dunnett's test. A $\chi^{2}$ test was used to determine the association between the clinical characteristics of the patients and the expression of ADRB2. Spearman's correlation test was used to evaluate the correlation between ADRB2 and sequestosome-1 (SQSTM1). The probability of differences in DFS was ascertained using the Kaplan-Meier method, with a log-rank test probe for significance. $\mathrm{P}<0.05$ was considered to indicate a statistically significant difference.

\section{Results}

Activation of ADRB2 by stress hormones promotes GC cell growth and survival. It is reported that catecholamines mediate the effects of stress on cells via adrenergic receptor activation. The present study first examined the effects of adrenergic receptor agonists on tumor growth and survival in GC cells. Three adrenergic receptor agonists, $10 \mu \mathrm{M}$ norepinephrine (a non-specific adrenergic receptor agonist), $5 \mu \mathrm{M}$ terbutaline (a specific ADRB2 agonist), $1 \mu \mathrm{M}$ xamoterol (a specific ADRB1 agonist), and $10 \mu \mathrm{M}$ propranolol (a non-specific $\beta$-adrenergic receptor antagonist) $(24,25)$ were used to treat the SGC-7901 and BGC-823 GC cell lines. The results showed that norepinephrine significantly promoted the proliferation of GC cells (Fig. 1A). A similar increase in cancer cell proliferation was observed with the ADRB2 agonist terbutaline, but not with theADRB1 agonist xamoterol. In addition, treating cells with norepinephrine and propranolol simultaneously inhibited the norepinephrine-induced increase in cancer cell proliferation (Fig. 1A). This indicated that $\beta 2$-adrenergic signaling was essential for the enhanced cell proliferation. To further identify the specific $\beta$-adrenergic receptor subtype responsible for catecholamine effects, tumor cell adrenoreceptor expression was inhibited using shRNA specific for human ADRB1 or ADRB2. The RT-qPCR method was used to determine which adrenergic receptor is present in GC cells and validate the knockdown efficiency. The data showed that ADRB1 and ADRB2 were expressed in SGC-7901 and BGC-823 cells. The RT-qPCR analysis indicated $\sim 80 \%$ downregulation at the mRNA level (Fig. 1A). In the PBS-treated GC cells, neither ADRB1-shRNA nor ADRB2-shRNA affected cell growth. In the norepinephrine-treated cells, ADRB2-shRNA significantly inhibited norepinephrine-induced tumor cell growth, but NC and ADRB1-shRNA failed to do so (Fig. 1A). Anchorage-dependent and anchorage-independent colony formation assays confirmed these observations (Fig. 1B and C). Taken together, these results suggest a critical role of ADRB2 in catecholamine-mediated GC cell proliferation.

Tumor cells develop resistance to apoptosis, allowing for their survival during stress. To evaluate the effect of catecholamine on GC cell survival, tumor cells cultured in serum-free medium were treated with adrenergic receptor agonists. Norepinephrine significantly protected tumor cells from nutrient deprivation (Fig. 2A and B). The ADRB2 

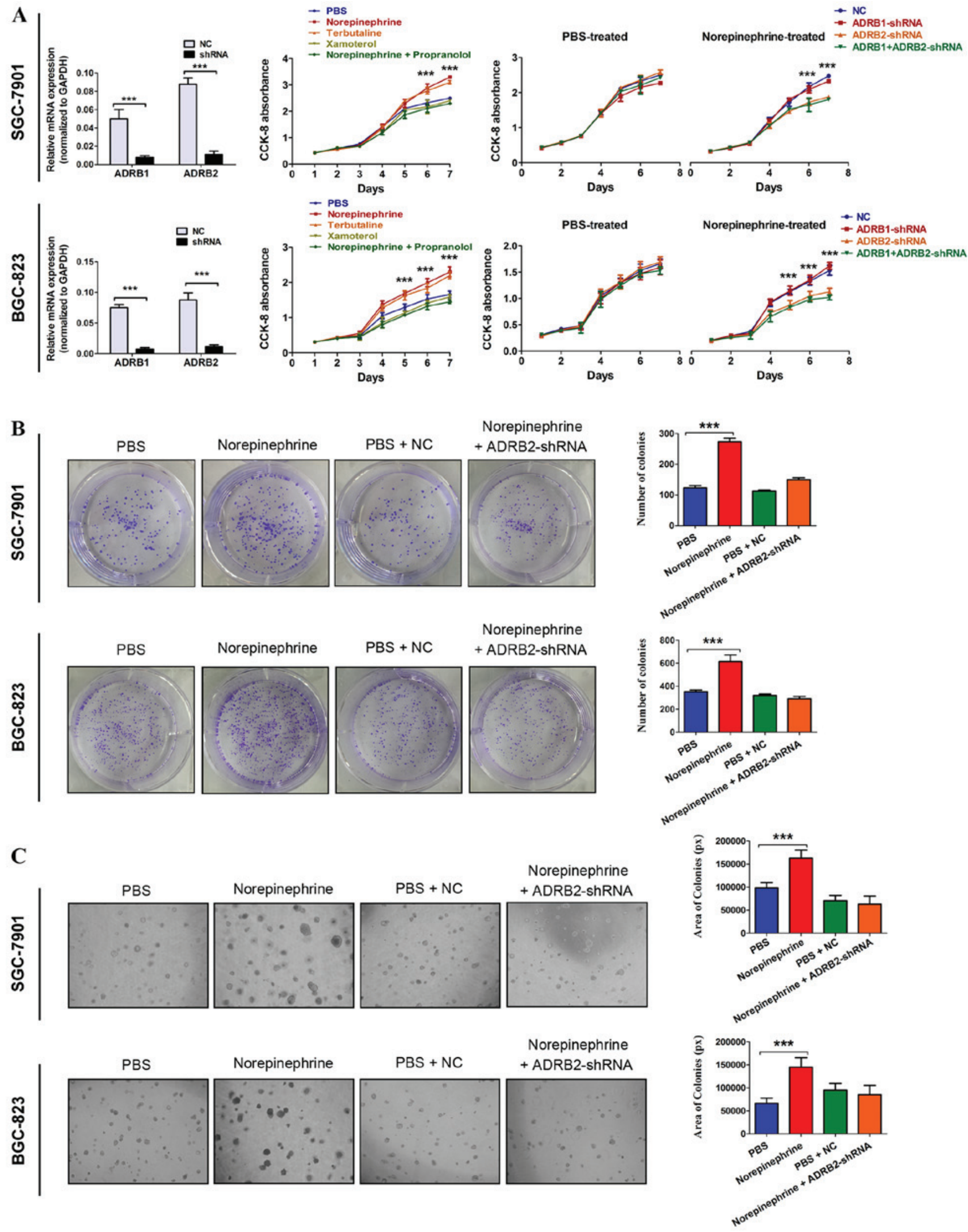

Figure 1. Activation of ADRB2 by stress hormone promotes GC cell growth. (A) Cell viability was determined using CCK-8 in GC cells treated with $10 \mu \mathrm{M}$ norepinephrine (a non-specific adrenergic receptor agonist), $5 \mu \mathrm{M}$ terbutaline (a specific ADRB2 agonist), $1 \mu \mathrm{M}$ xamoterol (a specific ADRB1 agonist), and $10 \mu \mathrm{M}$ propranolol (a non-specific $\beta$-adrenergic receptor antagonist). The significant P-values were derived from comparing the PBS group and norepinephrine/terbutaline group (left panels). Cell viability was determined using CCK-8 in ADRB1- or ADRB2-knockdown cells treated with $10 \mu \mathrm{M}$ norepinephrine. The significant P-values were derived from comparing the NC group and ADRB2-shRNA/ADRB1+ADRB2-shRNA group (right panels). Anchorage-dependent and anchorage-independent colony formation assays were used to determine the effect of norepinephrine and ADRB2 pathway on cell proliferation according to (B) colony number (magnification, x100) and (C) colony area (magnification, x200). Data represent the results from three independent experiments. ${ }^{* * *} \mathrm{P}<0.001$. GC, gastric cancer; ADRB2, $\beta 2$-adrenergic receptor; ADRB1, $\beta 1$-adrenergic receptor; CCK-8, Cell Counting Kit-8; shRNA, short hairpin RNA; NC, negative control. 
A
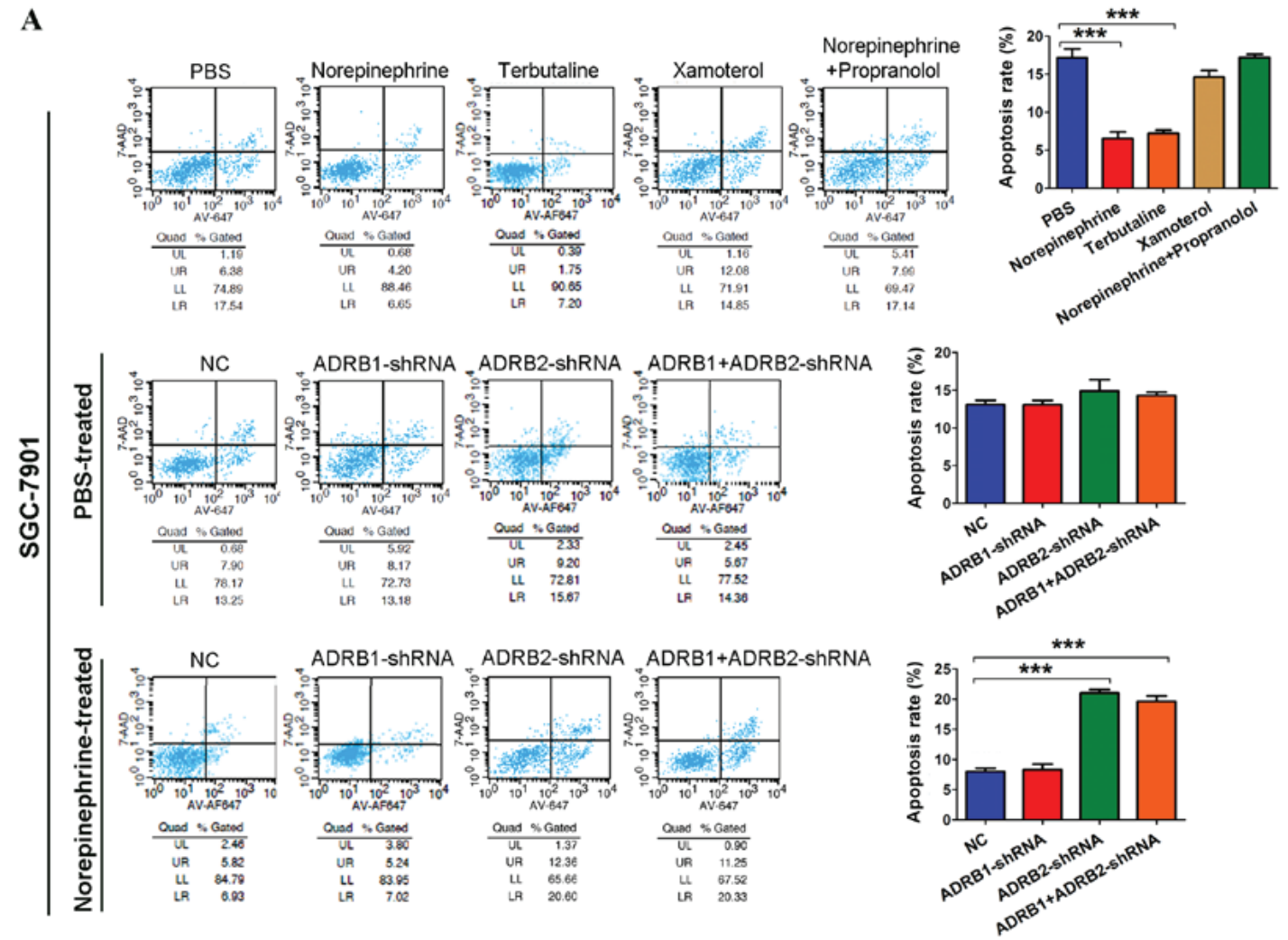

B
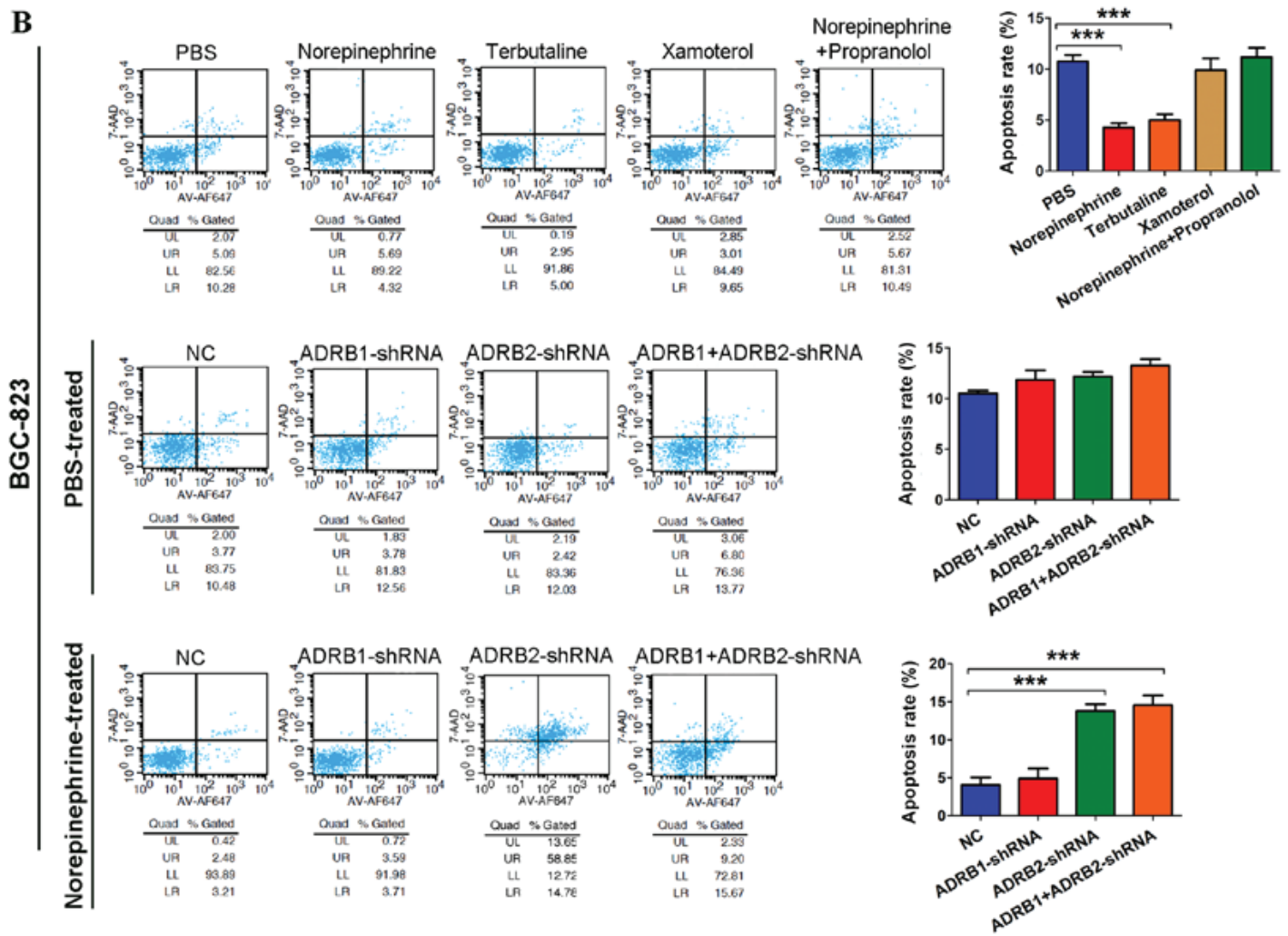

Figure 2. Activation of ADRB2 by stress hormone protects GC cells from nutrient limitation. (A) SGC-7901 and (B) BGC-823 GC cells were cultured in serum-free medium treated with adrenergic receptor agonists. The protective effect of norepinephrine on gastric cancer cells was also determined following ADRB1- or ADRB2-knockdown. Data represent the results from three independent experiments. ${ }^{* * * *} \mathrm{P}<0.001$. GC, gastric cancer; ADRB2, $\beta 2$-adrenergic receptor; ADRB1, $\beta 1$-adrenergic receptor; shRNA, short hairpin RNA; NC, negative control.

agonist terbutaline showed a similar effect on promoting tumor cell survival, but not the ADRB1 agonist xamoterol. In addition, propranolol inhibited the effects of norepinephrine.
Cells expressing ADRB1-shRNA and NC were protected from nutrient deprivation by norepinephrine, but cells expressing ADRB2-shRNA were not (Fig. 2A and B). Collectively, these 
A

\begin{tabular}{lll}
\hline NBS Norepinephrine & Norepinephrine \\
PBS + NC & + ADRB2-shRNA
\end{tabular}

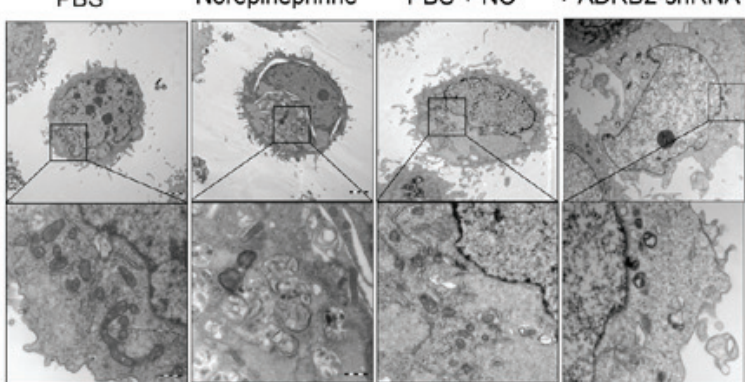

BGC-823

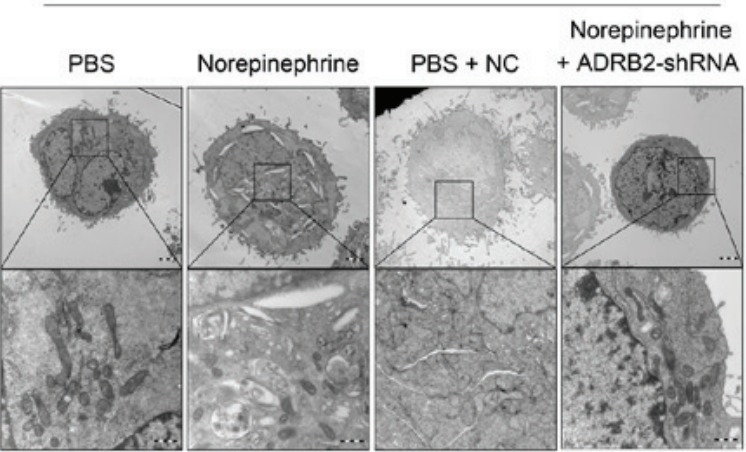

GC-7901

B

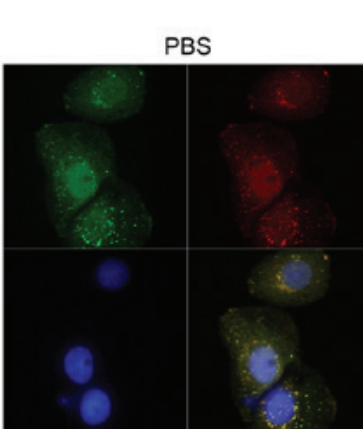

Norepinephrine

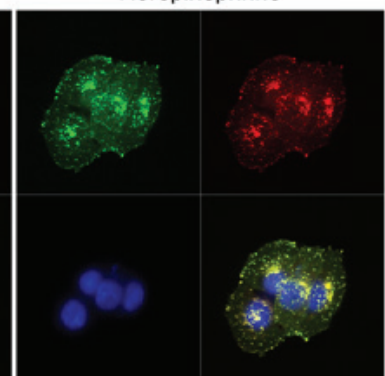

$\mathrm{PBS}+\mathrm{NC}$

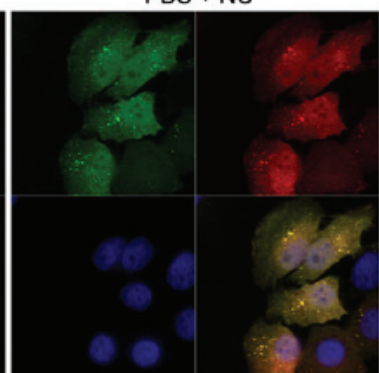

BGC-823
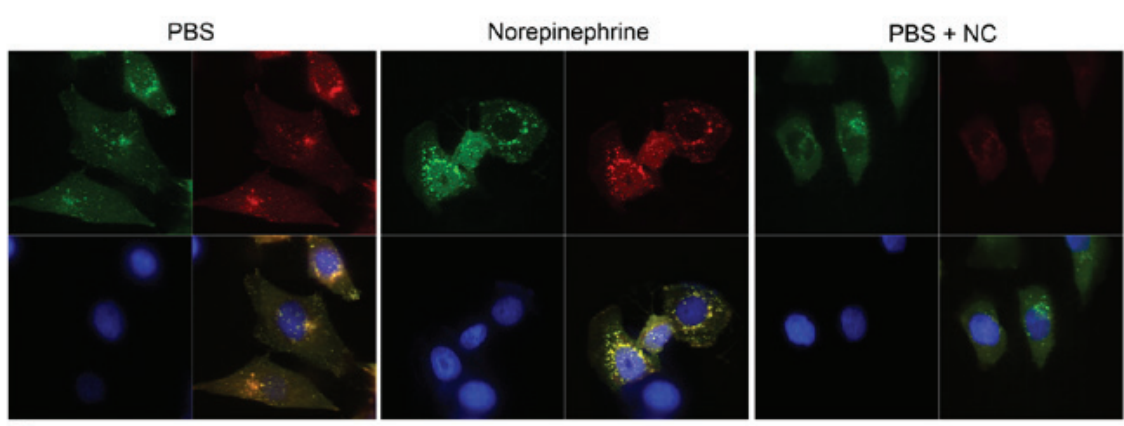

C

Red puncta $=$ Autolysosome
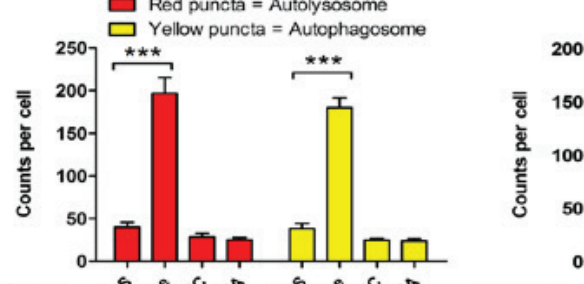

$\square$ Red puncta = Autolysosome

${ }^{200} \square$ Yellow puncta $=$ Autophagosome

D

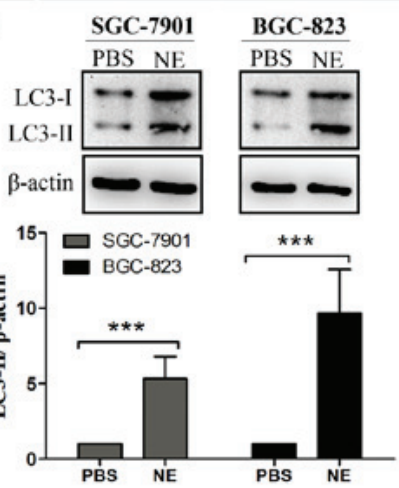

Figure 3. Activation of ADRB2 by norepinephrine leads to autophagy in GC cells. (A) Transmission electron microscope images of endogenic autophagic microstructure in SGC-7901 and BGC-823 cells show autophagosomes or autolysosomes that have a double-layer structure. Scale bar=500 nm. (B) Immunofluorescence analysis using GFP-mRFP-LC3 staining shows autophagosomes (yellow puncta) and autolysosomes (red puncta) (magnification, x600) (C) Numbers of LC3 puncta were quantified. (D) Western blot analysis of protein levels of LC3-I and LC3-II in cells confirmed autophagy formation following norepinephrine treatment. Data represent the results from three independent experiments. ${ }^{* * *} \mathrm{P}<0.001$. GC, gastric cancer; ADRB2, $\beta 2$-adrenergic receptor; shRNA, short hairpin RNA; NC, negative control; LC3, microtubule-associated protein 1 light chain 3.

results indicated that $\beta 2$-adrenergic signaling is essential for tumor cell survival under stress.

Specific activation of ADRB2 leads to autophagy in GC cells. One of the most prominent morphological features of cells treated with norepinephrine was the appearance of cytoplasmic vesicles. Transmission electron microscopy (TEM) showed that these vesicles were double-membrane vesicles containing cytoplasmic components, which is a hallmark of autophagy (Fig. 3A). To further confirm that the accumulation of 
autophagosome induced by norepinephrine was due to the specific activation of ADRB2, the cells were transfected with ADRB2-shRNA prior to norepinephrine treatment. The results revealed that the emergence of autophagosomes was markedly inhibited following transfection with ADRB2-shRNA (Fig. 3A). In addition to visually detecting autophagosomes by TEM, LC3-II is widely used as a marker of autophagy (26). Its lipidation and specific recruitment to autophagosomal membranes provides a shift from diffuse to punctate staining. In the present study, autophagic flux was monitored by exogenously introducing GFP-mRFP-LC3. As shown in Fig. $3 \mathrm{~B}$ and $\mathrm{C}$, norepinephrine treatment led to a significant increase in the number of GFP-LC3 puncta per cell, whereas shRNA-ADRB2 markedly inhibited the autophagy phenotype induced by norepinephrine. Consistently, norepinephrine treatment led to increased number of red puncta (autolysosomes) and yellow puncta (autophagosomes). These findings indicate that the accumulation of LC3-II induced by norepinephrine was due to increased autophagosome formation rather than the inhibition of autolysosomal degradation alone.

Given that the expression of exogenous LC3 can lead to false positives of LC3-II aggregation, the processing of endogenous LC3 in norepinephrine-treated cells was investigated by western blotting. LC3 lipidation was determined by the LC3-II ratio over $\beta$-actin instead of the LC3-II/LC3-I ratio as, during the initial stages of autophagy, LC3-II increases and LC3-I is subject to de novo synthesis, whereas the decrease of LC3-II at later stages is due to lysosomal degradation (27). The GC cells treated with norepinephrine exhibited a significant increase in the LC3-II/ $\beta$-actin ratio when compared with the control cells (Fig. 3D). To further confirm the effect of $\beta$-adrenergic receptor on LC3 lipidation, the LC3-II $/ \beta$-actin ratio was assessed following transfection with ADRB1-shRNA and ADRB2-shRNA in the GC cells. As shown in Fig. 4A, in the PBS-treated cells, neither ADRB1-shRNA nor ADRB2-shRNA affected the LC3-II/ $\beta$-actin ratio. However, in the norepinephrine treated cells, ADRB2-shRNA markedly inhibited norepinephrine-induced LC3 lipidation, whereas the NC and ADRB1-shRNA had no effect (Fig. 4A). In order to determine whether an increase in the number of autophagosomes result from an induction of the autophagic process, or from an arrest of lysosomal fusion/degradation, GC cells were treated with norepinephrine in the presence of E64d plus pepstain A, which is known to inhibit lysosomal acidic proteases and inhibit the degradation of LC3-II. Western blotting demonstrated an increased accumulation of LC3-II when lysosomal degradation was inhibited by E64d plus pepstain A (Fig. 4B), and treatment with NE plus lysosomal protease inhibitor increased LC3 accumulation compared with that in cells treated with lysosomal protease inhibitor alone. This is indicative of enhanced autophagosome formation induced by norepinephrine, rather than an impairment of autophagosome degradation. Taken together, these data suggest that ADRB2 signaling positively regulates autophagy in GC.

ADRB2-driven autophagy contributes to catecholamine-enhanced $G C$ cell proliferation and survival in vitro. Autophagy has two opposing functions in tumor cells in response to stress, the cytoprotective function and the cytotoxic function. The activation of ADRB2 in GC cells has been described as the regulation of autophagy. In order to evaluate the physiological effects of ADRB2-driven autophagy, cell proliferation and survival were monitored to discriminate whether these effects were cytoprotective or cytotoxic. Chloroquine was used to inhibit autophagy pharmacologically. The CCK- 8 assay showed that cell viability was further impaired by the inhibition of autophagy with chloroquine (Fig. 4C). Anchorage-dependent and anchorage-independent colony formation assays confirmed these findings (Fig. 4D and E). In addition, chloroquine treatment significantly attenuated the protective role of norepinephrine under nutrient deprivation (Fig. 4F). Collectively, the results indicate that ADRB2-driven autophagy serves a vital role in GC cell proliferation and survival.

Immobilization stress accelerates GC progression via ADRB2 signaling-induced autophagy in vivo. To investigate the effects of chronic stress on GC in vivo, SGC-7901 cells stably expressing luciferase were injected into BALB/c nude mice subcutaneously and orthotopically. The mice were then subjected to immobilization stress. Sustained chronic stress is known to elevate catecholamines and corticosteroid cortisol levels (28). Stress hormone levels were measured in the gastric tissues and plasma of these mice. As shown in Fig. 5A, samples derived from mice in the stress group had significantly higher stress hormone levels compared with mice in the control group. In the subcutaneous xenograft mouse model, after 20 days, the mice in the stress group exhibited a marked increase in tumor size compared with mice in the control group (Fig. 5B-D). Similar results were observed in the orthotopic xenograft model (Fig. 5E and F). The mechanisms through which chronic behavioral stress promotes GC progression were investigated. ADRB2-driven autophagy was found to promote catecholamine-enhanced GC cell proliferation and survival in vitro. Subsequently, whether the activation of $\beta$-adrenergic signaling similarly induces xenograft tumor growth of GC cells by unregulating autophagy was investigated. Propranolol, a $\beta$-adrenergic receptor antagonist, was used to inhibit $\beta$-adrenergic signaling, and ADRB2-shRNA was used to knockdown the expression of ADRB2. As shown in Fig. 5B-F, propranolol treatment and ADRB2-shRNA transduction significantly inhibited chronic stress-induced GC progression. Furthermore, inhibition of autophagy using chloroquine attenuated chronic stress-induced GC progression. Immunohistochemical staining of Ki-67 and a terminal-deoxynucleoitidyl transferase mediated nick end labeling (TUNEL) assay was used to determine cell proliferation and survival in mice, respectively. Ki-67 staining was elevated in tumors from mice in the stress group, however propranolol treatment, ADRB2-shRNA transfection and chloroquine treatment markedly attenuated this effect (Fig. 5G). The TUNEL assays showed that chronic stress reduced apoptosis compared with that in the control group, whereas propranolol treatment, ADRB2-shRNA transfection and chloroquine treatment inhibited this effect (Fig. 5G). These results suggest that the activation of autophagy induced by the $\beta 2$-adrenergic signaling pathway promoted tumor growth in GC xenograft mouse models.

AMPK-ULK1 pathway is required for autophagy in response to ADRB2 signaling activation. The mechanisms underlying 
A

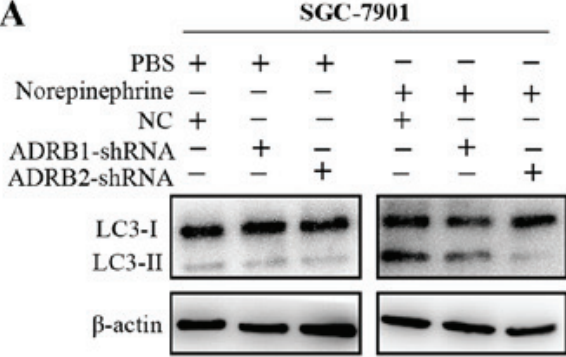

B

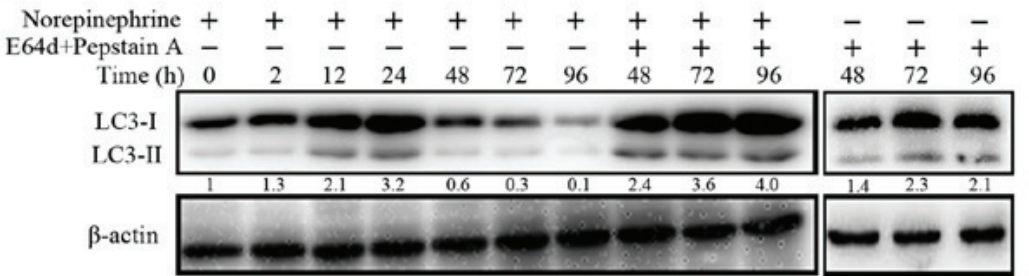

$\mathrm{C}_{\text {SGC-7901 }} \rightarrow$ PBS

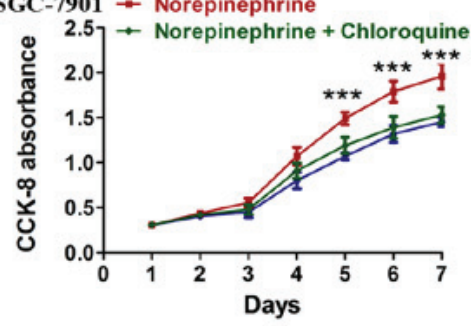

$\rightarrow$ PBS

BGC-823 - Norepinephrine

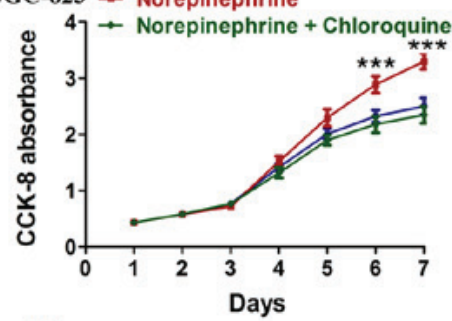

D
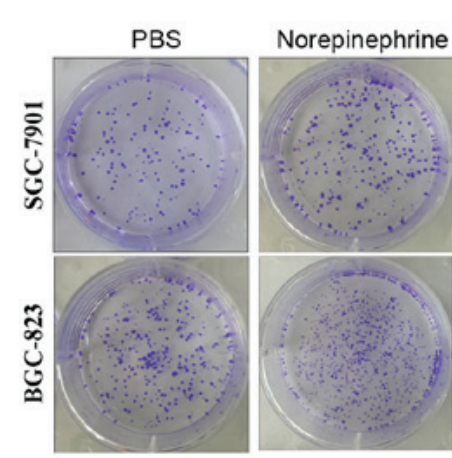

Norepinephrine

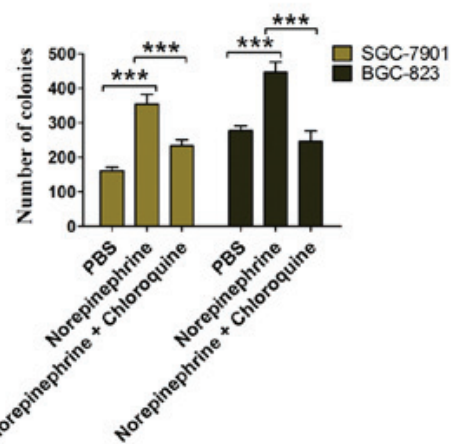

$\mathbf{E}$
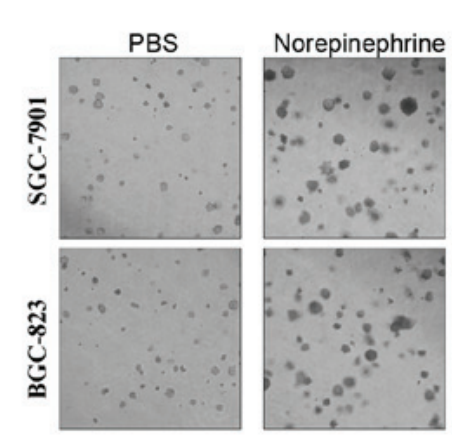

Norepinephrine
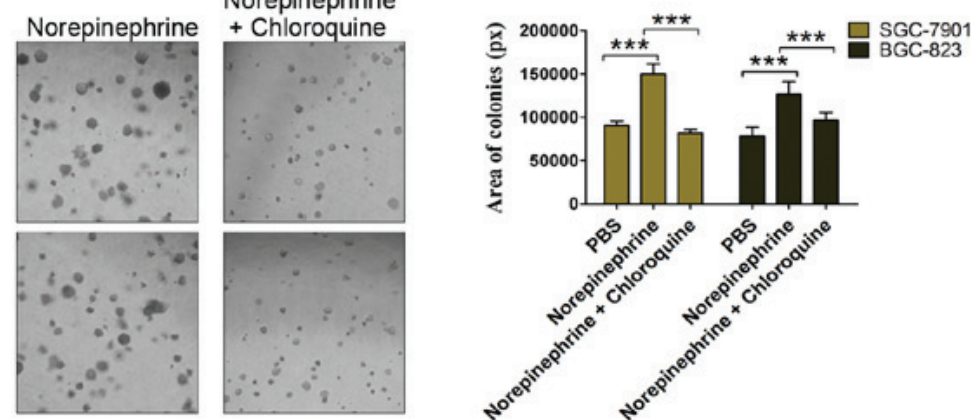

$\mathbf{F}$
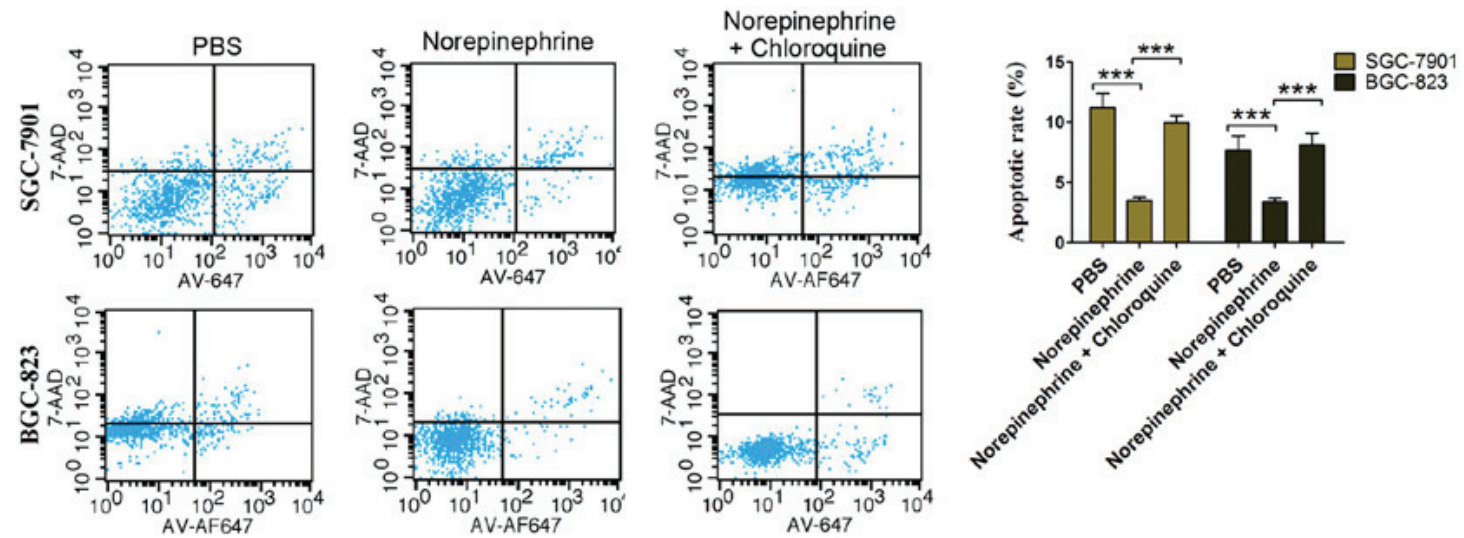

Figure 4. ADRB2-driven autophagic flux contributes to catecholamine-enhanced GC cell proliferation and anti-apoptosis. (A) Protein levels of LC3-I and LC3-II were detected in ADRB1- or ADRB2-knockdown cells. Cells were treated with norepinephrine for 24 h. (B) Autophagic flux was monitored by dynamic western blotting in SGC-7901 cells. Cell proliferation was determined using a (C) CCK-8 assay (The significant P-values were derived from comparing the PBS group and norepinephrine group.), and anchorage-dependent and anchorage-independent colony formation assays determining (D) colony number (magnification, $\mathrm{x} 100$ ) and (E) area (magnification, $\mathrm{x} 200$ ) following the inhibition of autophagy with chloroquine. (F) Cell apoptosis was determined using flow cytometry in cells cultured in serum-free medium. Data represent the results from three independent experiments. ${ }^{* * *} \mathrm{P}<0.001$. GC, gastric cancer; ADRB2, 32 -adrenergic receptor; shRNA, short hairpin RNA; NC, negative control; LC3, microtubule-associated protein 1 light chain 3. 

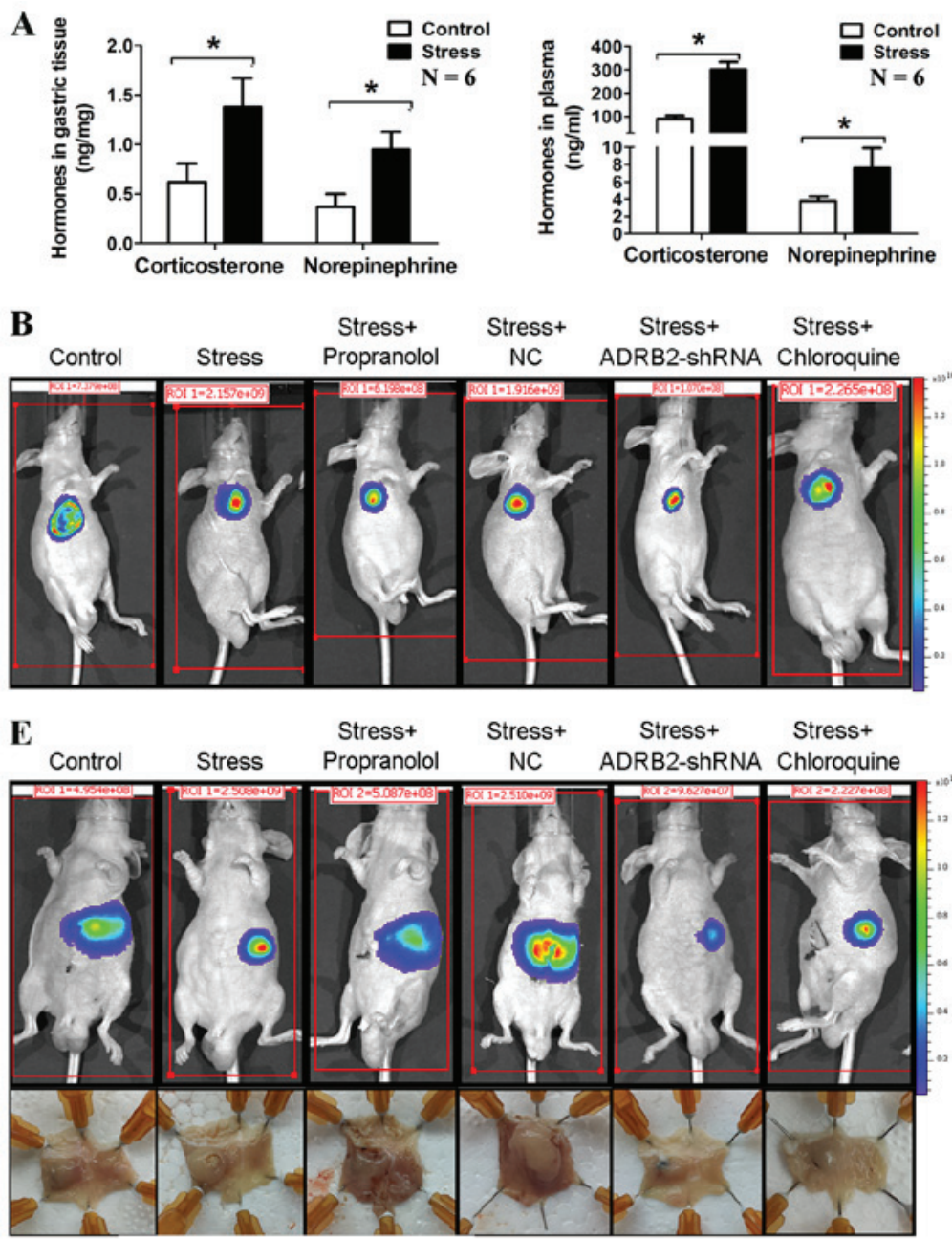

G
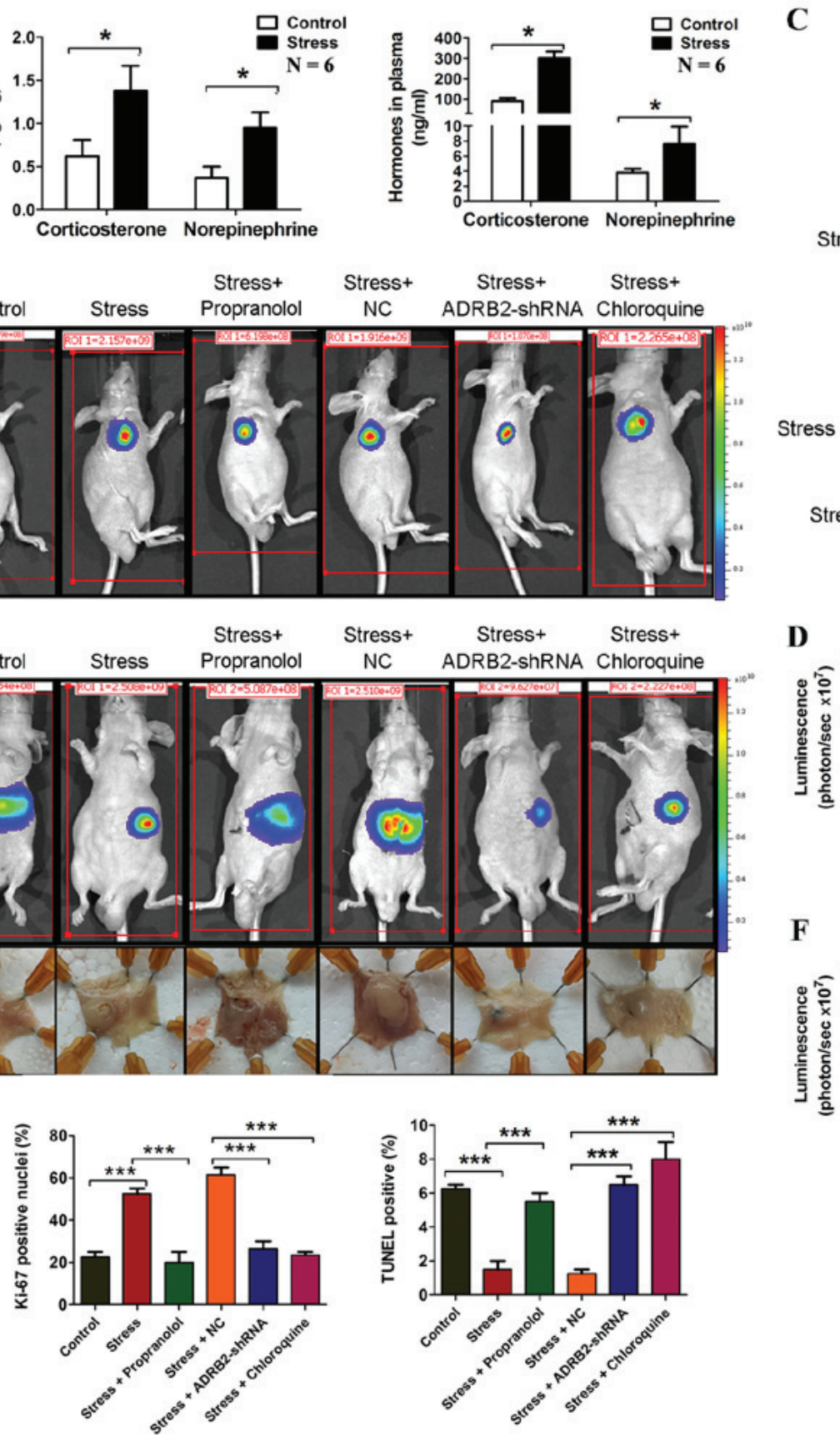

Stress + Propranolol
Stress + NC

Stress + ADRB2-shRNA

Stress + Chloroquine
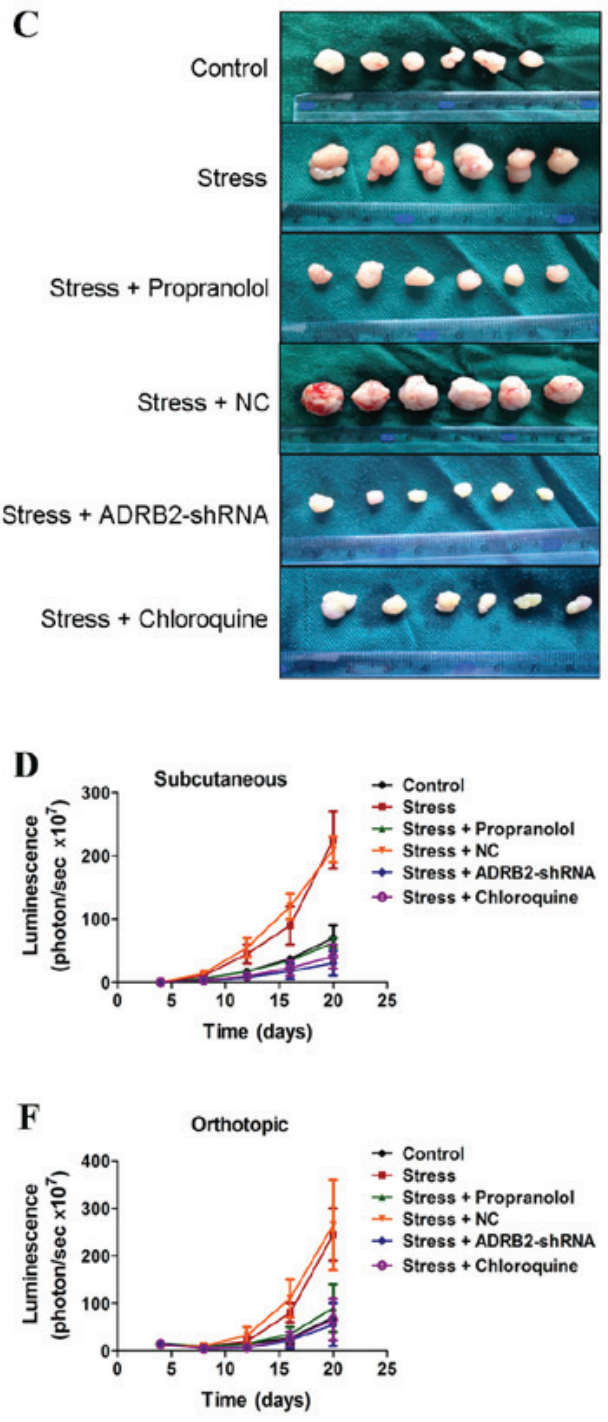
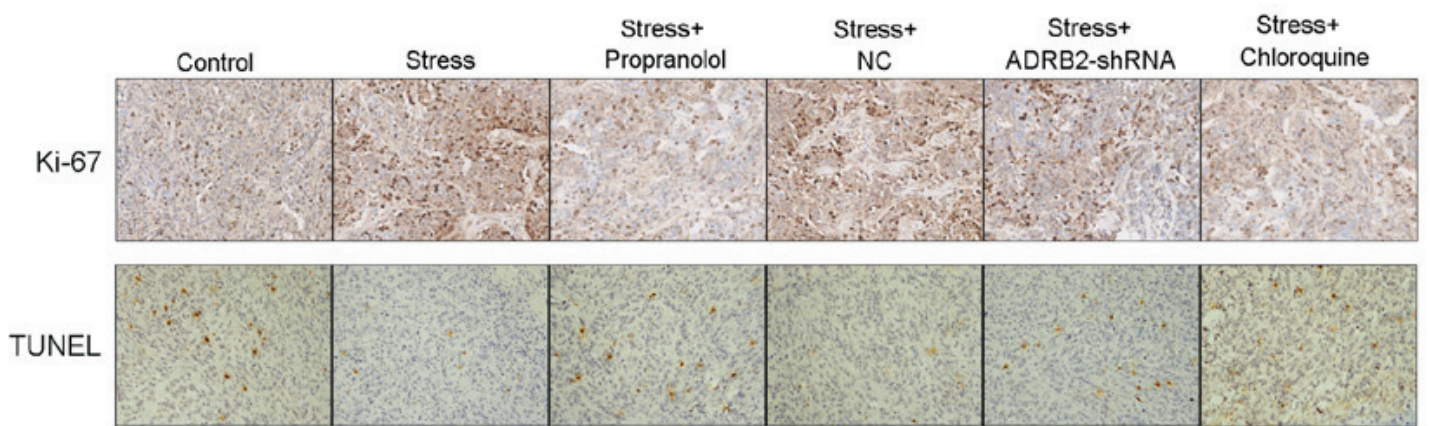

Figure 5. Immobilization stress accelerates GC progression via ADRB2 signaling-induced autophagy in vivo. (A) Concentrations of stress hormones (norepinephrine and corticosterone) were determined in gastric tissue and plasma samples with high performance liquid chromatography. SGC-7901 cells stably expressing luciferase were injected into BALB/c nude mice subcutaneously. Alzet osmotic minipumps containing PBS or $2 \mathrm{mg} / \mathrm{kg} / \mathrm{d}$ propranolol hydrochloride were inserted on the nape of neck 7 days prior to initiation of restraint stress. Representative images of (B) tumors and (C) mice at the end-point and (D) tumor growth curves are shown. The xenografts were transplanted in the gastric wall by inoculating tumor fragments $(2 \times 2 \times 2 \mathrm{~mm})$ through laparotomy. (E) Representative images of mice and tumors at the end-point and (F) tumor growth curves are shown. (G) Immunohistochemical staining of Ki-67 and a TUNEL assay were adapted to determine the molecular markers of cell proliferation and anti-apoptosis in orthotopic transplantation mice (magnification, x200). Data represent the results from three independent experiments. ${ }^{*} \mathrm{P}<0.05$ and ${ }^{* * *} \mathrm{P}<0.001$. GC, gastric cancer; ADRB2, $\beta 2$-adrenergic receptor; shRNA, short hairpin RNA; NC, negative control; TUNEL, terminal-deoxynucleoitidyl transferase mediated nick end labeling. 
A

SGC-7901

Norepinephrine $-\frac{\mathrm{NC}}{-}+\frac{\text { ADRB2-shRNA }}{-+} \frac{\mathrm{NC}}{-+} \frac{\text { ADRB2-shRNA }}{-+}$

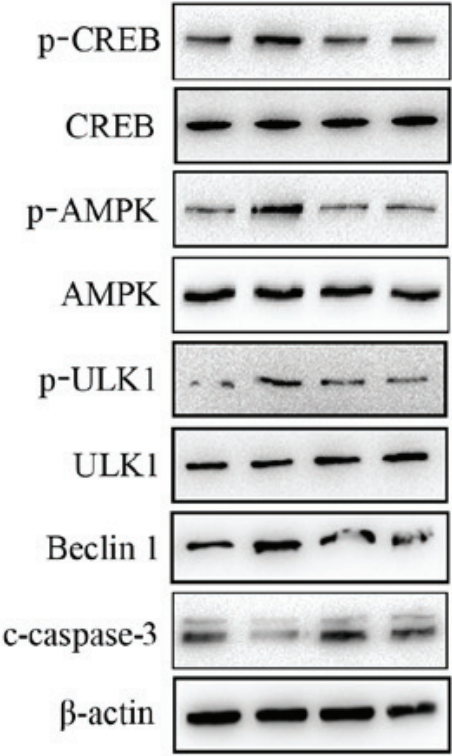

C
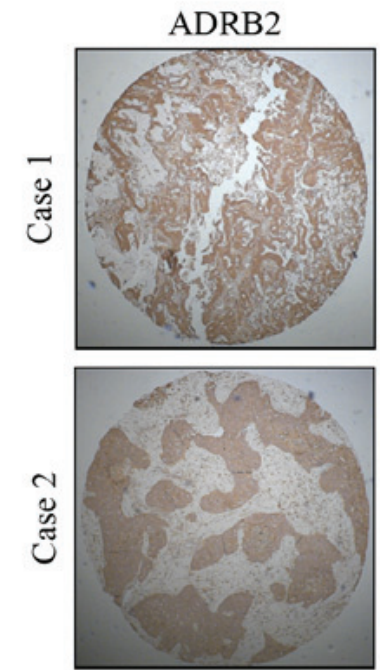

D

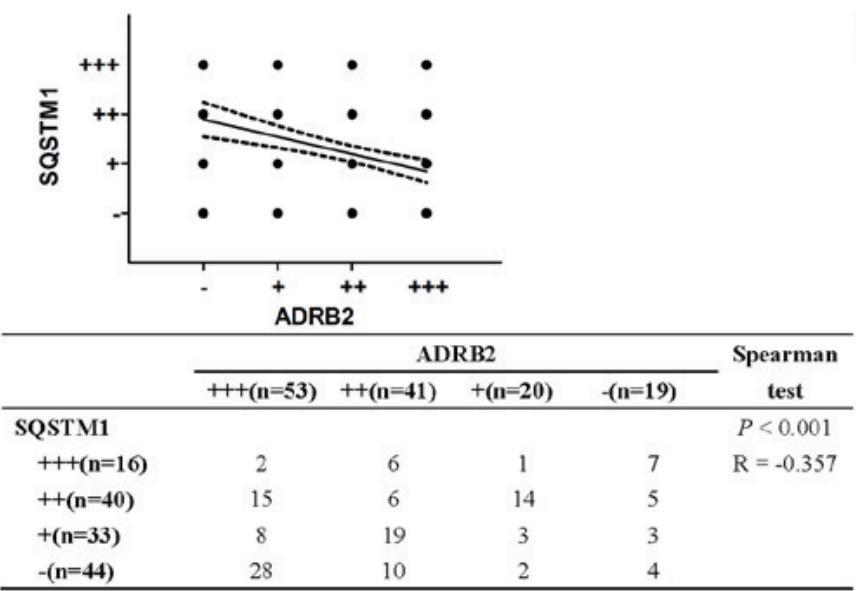

$\mathbf{E}$

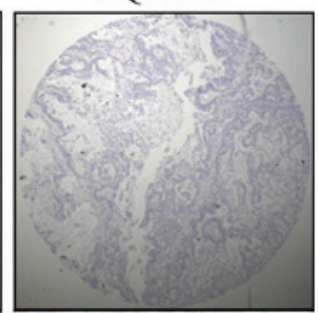

F
B

Norepinephrine - +-+
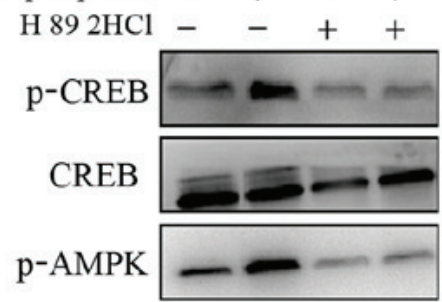

AMPK

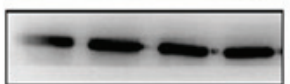

p-ULK1

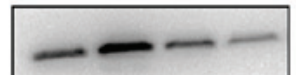

ULK1

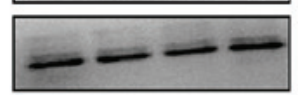

$\beta$-actin

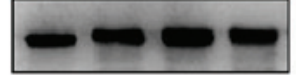

BGC-823

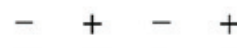

$-\quad+\quad+$
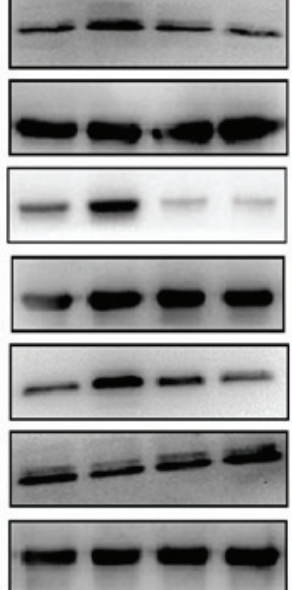
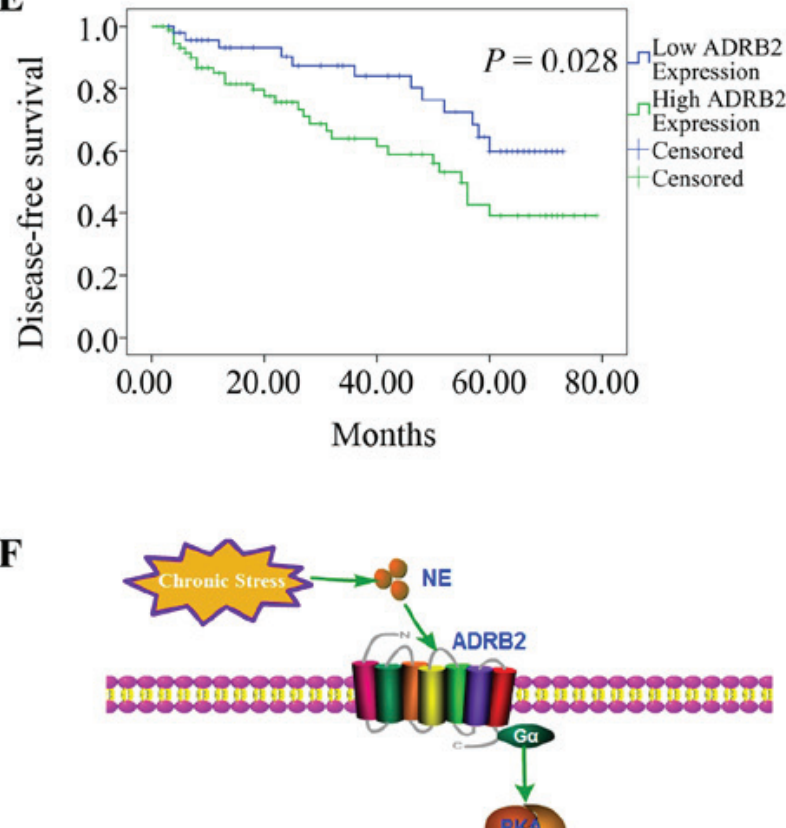

Figure 6. Activation of theAMPK-ULK1 pathway is critical for autophagy induced by norepinephrine. (A) Expression levels of AMPK-ULK1 pathway proteins were detected by western blotting. (B) SGC-7901 and BGC-823 cells were treated with norepinephrine with or without $\mathrm{H} 892 \mathrm{HCl}(50 \mathrm{nM})$, and the expression levels of AMPK-ULK1 pathway proteins were detected with western blotting. (C) Representative images of the expression of ADRB2 and SQSTM1 in the gastric cancer tissue microarray (magnification, x100). (D) Correlation between the expression of ADRB2 and SQSTM1 was examined by Spearman's correlation test. (E) Survival analysis of patients with gastric cancer with high ADRB2 expression and low ADRB2 expression. (F) Schematic representation of the proposed mechanism. Data represent the results from three independent experiments. ADRB2, $\beta 2$-adrenergic receptor; shRNA, short hairpin RNA; NC, negative control AMPK, adenosine 5'-monophosphate (AMP)-activated protein kinase; CREB, cAMP-response element binding protein; p, phosphorylated; SQSTM1, sequestosome-1; ULK1, unc-51 like autophagy activating kinase 1; PKA, protein kinase A; NE, norepinephrine. 
norepinephrine-mediated GC cell autophagy and survival were subsequently investigated. CREB is a key downstream effector of the $\beta$-adrenergic signaling pathway (29). In line with this finding, treatment with norepinephrine promoted CREB activation in GC cells and this effect was inhibited by ADRB2 silencing (Fig. 6A). AMPK has been demonstrated to induce autophagy by activating ULK1 (30). The AMPK-ULK1 pathway was activated by norepinephrine treatment (Fig. 6A), and knockdown of ADRB2 significantly attenuated the activation of AMPK-ULK1 induced by norepinephrine (Fig. 6A). Beclin1 serves an important role in the initiation of autophagosome formation. The expression levels of Beclin1 were analyzed by western blotting. As shown in Fig. 6A, norepinephrine treatment markedly increased the protein levels of Beclin1, but had no effect in ADRB2-depleted cells (Fig. 6A). Additionally, norepinephrine treatment markedly decreased the apoptosis marker, cleaved-caspase-3, whereas norepinephrine had no effect on the levels of cleaved-caspase-3 in ADRB2-knockdown cells (Fig. 6A). $\mathrm{H} 892 \mathrm{HCl}$, an inhibitor of protein kinase $\mathrm{A}$, abrogated the NE-induced phosphorylation of AMPK and ULK1 (Fig. 6B). Collectively these results suggest that autophagy induced by norepinephrine was attributed to activation of the AMPK-ULK1 pathway.

Association of ADRB2 with SQSTM1 in clinical GC samples. To investigate the clinical relevance of the above findings, immunohistochemical staining was performed to determine the protein expression levels of ADRB2 in $133 \mathrm{GC}$ tumor samples (Table I). High protein levels of ADRB2 were positively were associated with the TNM stage, indicating that high protein levels of ADRB2 may serve as a predictor for staging. High protein levels of ADRB2were also associated with poor prognosis in patients with GC (Fig. 6E). The p62/SQSTM1 protein serves as a link between LC3 and ubiquitinated substrates and is degraded in autolysosomes, indicating that the protein levels of p62/SQSTM1 reflect the autophagic status (31). Therefore, the present study investigated the correlation between ADRB2 and autophagic marker p62/SQSTM1 in GC tumor samples. TMA showed that there was a negative correlation between the expression levels of ADRB2 and p62/SQSTM1(Fig. 6C and D). Taken together, the results of the present study demonstrate the positive effect of autophagy on norepinephrine-induced GC progression and revealed an important regulatory mechanism of ADRB2 signaling activation in autophagy (Fig. 6F).

\section{Discussion}

Emerging evidence has implicated that chronic stress, specifically adrenergic signaling, is involved in the onset and progression of various types of cancer $(32,33)$. The stomach is one of the most susceptible target organs of chronic stress, and patients with GC experiencing severe psychological distress at the time of diagnosis frequently experience prior psychological stress (34). However, limited reports of the association between stress and gastric cancer have been published. Molecular insights into how chronic stress affects gastric tumorigenesis may offer novel treatment options. The results presented in the present study is the first, to the best of our knowledge, to provide direct preclinical evidence for the role of chronic stress in the progression of GC.

The present study found that chronic stress increased norepinephrine and corticosterone levels in plasma and gastric tissues, and markedly enhanced GC development, as demonstrated in subcutaneous and orthotopic xenograft mouse models. Previous studies have demonstrated that increased levels of circulating catecholamines, particularly norepinephrine, serve a crucial role in cancer development. In the present study, it was demonstrated that mice implanted with an osmotic pump that releases physiologically relevant doses of norepinephrine enhanced the progression of GC. The mechanism by which chronic stress promotes gastric tumorigenesis remains to be fully elucidated. Previous studies have shown that chronic stress induces cancer initiation and development by impairing the immune system, which is essential for immune surveillance. These immune dysfunctions include cytotoxicity in natural killer cells, cytokine production and $\mathrm{T}$ cell mitogenesis (35). However, chronic stress enhances tumor development in immunodeficient nude mice. This indicates that additional mechanisms are involved in chronic stress induced tumor development. The present study demonstrated that the norepinephrine-induced activation of autophagy in gastric cancer cells was responsible for the progression of GC.

Autophagy is an important cellular process that serves a critical role in tumor initiation and progression. However, there is a lack of information regarding the effects of chronic stress on gastric cancer cell autophagy. In the present study, it was demonstrated that stress hormone norepinephrine triggers gastric cancer cell autophagy. The induction of autophagy was a novel finding of $\beta 2$-adrenergic activation in GC cells, as demonstrated by the appearance of double-membrane vesicles, the punctuate of GFP-RFP-LC3 distribution in the cytoplasm and the corresponding increase in the LC3-II/ $/$-actin ratios. Additionally, autophagic flux was monitored by protein degradation assays. Enhanced autophagosome formation was induced by norepinephrine, rather than the impairment of autophagosome degradation alone. As autophagy is involved in the degradation of proteins, the correlation between ADRB2 and autophagic marker p62/SQSTM1 protein was investigated in GC tumor samples to determine its clinical relevance. The TMA showed a negative correlation between the expression of ADRB2 and p62/SQSTM1.

However, there is contradictory information with regards to whether autophagy is a tumor promoter or tumor suppressor. Under conditions of severe stress, autophagy increases cell death (36), whereas, in certain instances, autophagy formation is necessary for maintaining normal physiology (11). The underlying question with regards to autophagy in gastric cancer is whether its role is detrimental or protective. The present study is the first, to the best of our knowledge, to demonstrate the protective effects of autophagy in norepinephrine-induced GC progression in vitro and in vivo; the results shed light on the importance of autophagy regulation in the development of GC.

The signaling pathway downstream of stress-activated ADRB2 for regulating autophagy remains to be elucidated. The present study demonstrated that silencing ADRB2 reduced the autophagy-related gene Beclin1 and impaired cell survival via 
inactivation of the AMPK-ULK1 pathway. It is established that AMPK serves a major role in autophagy and is activated by the increase of cellular AMP/ATP ratios. This in-turn favors the binding of adenine nucleotides to the $\gamma$ subunit of AMPK (37). Previously, AMPK was reported to be activated by protein kinase A (38). The present study demonstrated that the knockdown of ADRB2 significantly attenuated the activation of AMPK-ULK1 pathway induced by norepinephrine. This indicates that the knockdown of ADRB2 may reduce the expression of autophagy-related genes, including Beclin1, and inactivate the autophagic AMPK-ULK1 pathway, which in turn, attenuates autophagy and inhibits growth of human GC cells. High protein levels of ADRB2 correlated positively with TNM stage and predicted an unfavorable prognosis. A recent meta-analysis also demonstrated that non-selective $\beta$-blockers have the potential to decrease tumor incidence by targeting the $\beta$-adrenergic receptor. Therefore, $\beta$-adrenergic receptors, specifically ADRB2, may be a promising therapeutic target for cancer treatment and prevention.

In conclusion, based on the subcutaneous and orthotopic xenograft mouse models, the present study is the first, to the best of our knowledge, to provide preclinical evidence of the role of chronic stress in the progression of GC. The novel mechanism proposed outlines the chronic stress-mediated development of GG cancer. Insights from the present study advance current understanding of the mechanisms involved in chronic stress, $\beta$-adrenergic signaling, autophagy regulation and cancer progression. Due the efficacy of $\beta 2$-adrenergic modulation on GC inhibition, the present study supports the potential use of an adrenoceptor antagonist, such as propranolol, for the treatment of GC.

\section{Acknowledgements}

Not applicable.

\section{Funding}

This study was supported by the National Natural Science Foundation of China (grant no. 81702369), the China Postdoctoral Science Foundation (grant nos. 2018T110534 and 2016M601868), the Postdoctoral Science Foundation of Jiangsu Province (grant no. 1701033B), the National Natural Science Foundation of China (grant no. 81572362), the National Natural Science Foundation Project of International Cooperation (grantno.81361120398), the Primary Research and Development Plan of Jiangsu Province (grant no. BE2016786), the Program for Development of Innovative Research Team at the First Affiliated Hospital of NJMU; the Priority Academic Program Development of Jiangsu Higher Education Institutions (grant no. JX10231801), the 333 Project of Jiangsu Province (grant no. BRA2015474), Jiangsu Key Medical Discipline (General Surgery) and Jiangsu Key Lab of Cancer Biomarkers, Prevention and Treatment, Collaborative Innovation Center for Cancer Personalized Medicine, Nanjing Medical University.

\section{Availability of data and materials}

All data generated or analysed during this study are included in this published article.

\section{Authors' contributions}

$\mathrm{XZ}, \mathrm{BL}, \mathrm{ZL}$ and $\mathrm{JZ}$ performed the experiments, acquired the data and drafted the manuscript. JY and LZ collected the tissue samples. ZX analyzed and interpreted the data. All authors have read and approved the final manuscript and agreed to be accountable for all aspects of the research in ensuring that the accuracy or integrity of any part of the work are appropriately investigated and resolved.

\section{Ethics approval and consent to participate}

All applicable international, national, and/or institutional guidelines for the care and use of animals were followed. Animal research was approved by the Animal Care and Use Committee of the Laboratory Animal Center of Nantong University. All procedures performed in experiments involving human participants were in accordance with the ethical standards of the institutional and/or national research committee and with the 1964 Helsinki declaration and its later amendments or comparable ethical standards. The present study was approved by the Institutional Ethical Board of the First Affiliated Hospital of Nanjing Medical University. Informed consent was obtained from all individual participants included in the study.

\section{Patient consent for publication}

Not applicable.

\section{Competing interests}

The authors declare that they have no competing interests.

\section{References}

1. Chida Y, Hamer M, Wardle J and Steptoe A: Do stress-related psychosocial factors contribute to cancer incidence and survival? Nat Clin Pract Oncol 5: 466-475, 2008.

2. Ng BH and Tsang HW: Psychophysiological outcomes of health qigong for chronic conditions: A systematic review. Psychophysiology 46: 257-269, 2009.

3. Bulli F, Miccinesi G, Maruelli A, Katz M and Paci E: The measure of psychological distress in cancer patients: the use of Distress Thermometer in the Oncological Rehabilitation Center of Florence. Support Care Cancer 17: 771-779, 2009.

4. Moussas GI, Papadopoulou AG, Christodoulaki AG and Karkanias AP: Psychological and psychiatric problems in cancer patients: relationship to the localization of the disease. Psychiatriki 23: 46-60, 2012 (In Greek).

5. Shan T, Cui X, Li W, Lin W, Li Y, Chen X and Wu T: Novel regulatory program for norepinephrine-induced epithelial-mesenchymal transition in gastric adenocarcinoma cell lines. Cancer Sci 105: 847-856, 2014.

6. Moretti S, Massi D, Farini V, Baroni G, Parri M, Innocenti S, Cecchi R and Chiarugi P: $\beta$-adrenoceptors are upregulated in human melanoma and their activation releases pro-tumorigenic cytokines and metalloproteases in melanoma cell lines. Lab Invest 93: 279-290, 2013.

7. Hassan S, Karpova Y, Baiz D, Yancey D, Pullikuth A, Flores A, Register T, Cline JM, D'Agostino R Jr, Danial N, et al: Behavioral stress accelerates prostate cancer development in mice. J Clin Invest 123: 874-886, 2013.

8. Armaiz-Pena GN, Allen JK, Cruz A, Stone RL, Nick AM, Lin YG, Han LY, Mangala LS, Villares GJ, Vivas-Mejia P, et al: Src activation by $\beta$-adrenoreceptors is a key switch for tumour metastasis. Nat Commun 4: 1403, 2013. 
9. Thaker PH,Han LY, Kamat AA, Arevalo JM, Takahashi R, Lu C, Jennings NB, Armaiz-Pena G, Bankson JA, Ravoori M, et al: Chronic stress promotes tumor growth and angiogenesis in a mouse model of ovarian carcinoma. Nat Med 12: 939-944, 2006.

10. Konturek SJ, Brzozowski T, Konturek PC, Zwirska-Korczala K and Reiter RJ: Day/night differences in stress-induced gastric lesions in rats with an intact pineal gland or after pinealectomy. J Pineal Res 44: 408-415, 2008.

11. Gewirtz DA: The four faces of autophagy: Implications for cancer therapy. Cancer Res 74: 647-651, 2014.

12. Lozy F and Karantza V: Autophagy and cancer cell metabolism. Semin Cell Dev Biol 23: 395-401, 2012.

13. Mizushima N and Komatsu M: Autophagy: Renovation of cells and tissues. Cell 147: 728-741, 2011.

14. Levine B and Kroemer G: Autophagy in aging, disease and death: The true identity of a cell death impostor. Cell Death Differ 16: 1-2, 2009.

15. Farah BL, Sinha RA, Wu Y, Singh BK, Zhou J, Bay BH and Yen PM: $\beta$-adrenergic agonist and antagonist regulation of autophagy in HepG2 cells, primary mouse hepatocytes, and mouse liver. PLoS One 9: e98155, 2014.

16. Lizaso A, Tan KT and Lee YH: $\beta$-adrenergic receptor-stimulated lipolysis requires the RAB7-mediated autolysosomal lipid degradation. Autophagy 9: 1228-1243, 2013.

17. Funakoshi T, Aki T, Unuma K and Uemura K: Lysosome vacuolation disrupts the completion of autophagy during norephedrine exposure in SH-SY5Y human neuroblastoma cells. Brain Res 1490: 9-22, 2013.

18. Eskelinen EL: The dual role of autophagy in cancer. Curr Opin Pharmacol 11: 294-300, 2011.

19. Dikken JL, van de Velde CJ, Gönen M, Verheij M, Brennan MF and Coit DG: The New American Joint Committee on Cancer/International Union Against Cancer staging system for adenocarcinoma of the stomach: Increased complexity without clear improvement in predictive accuracy. Ann Surg Oncol 19: 2443-2451, 2012

20. Meng D, Chen Y, Zhao Y, Wang J, Yun D, Yang S, Chen J, Chen H and Lu D: Expression and prognostic significance of TCTN1 in human glioblastoma. J Transl Med 12: 288, 2014.

21. Wang S, Wu X, Chen Y,Zhang J, Ding J,Zhou Y,HeS, Tan Y, Qiang F, Bai J, et al:: Prognostic and predictive role of JWA and XRCC1 expressions in gastric cancer. Clin Cancer Res 18: 2987-2996, 2012.

22. Livak KJ and Schmittgen TD: Analysis of relative gene expression data using real-time quantitative PCR and the 2(-Delta Delta C(T)) Method. Methods 25: 402-408, 2001.

23. Zamora-González EO, Santerre A, Palomera-Avalos V and Morales-Villagrán A: A chronic combinatory stress model that activates the HPA axis and avoids habituation in BALB/C mice. J Neurosci Methods 213: 70-75, 2013.

24. Bernabé DG, Tamae AC, Biasoli ER and Oliveira SH: Stress hormones increase cell proliferation and regulates interleukin-6 secretion in human oral squamous cell carcinoma cells. Brain Behav Immun 25: 574-583, 2011.

25. Nilsson MB, Armaiz-Pena G, Takahashi R, Lin YG, Trevino J, Li Y, Jennings N, Arevalo J, Lutgendorf SK, Gallick GE, et al: Stress hormones regulate interleukin- 6 expression by human ovarian carcinoma cells through a Src-dependent mechanism. J Biol Chem 282: 29919-29926, 2007.
26. Klionsky DJ, Abdelmohsen K, Abe A, Abedin MJ, Abeliovich H, Acevedo Arozena A, Adachi H, Adams CM, Adams PD, Adeli K, et al: Guidelines for the use and interpretation of assays for monitoring autophagy (3rd edition). Autophagy 12: 1-222, 2016.

27. Wu FQ, Fang T, Yu LX, Lv GS, Lv HW, Liang D, Li T, Wang CZ, Tan YX, Ding J, et al: ADRB2 signaling promotes HCC progression and sorafenib resistance by inhibiting autophagic degradation of HIF1 $\alpha$. J Hepatol 65: 314-324, 2016.

28. Hulsurkar M, Li Z, Zhang Y, Li X, Zheng D and Li W: Beta-adrenergic signaling promotes tumor angiogenesis and prostate cancer progression through HDAC2-mediated suppression of thrombospondin-1. Oncogene 36: 1525-1536, 2017.

29. Hayakawa Y, Sakitani K, Konishi M, Asfaha S, Niikura R, Tomita H, Renz BW, Tailor Y, Macchini M, Middelhoff $\mathrm{M}$, et al: Nerve growth factor promotes gastric tumorigenesis through aberrant cholinergic signaling. Cancer Cell 31: 21-34, 2017.

30. Xie CM, Liu XY, Sham KW, Lai JM and Cheng CH: Silencing of EEF2K (eukaryotic elongation factor-2 kinase) reveals AMPK-ULK1-dependent autophagy in colon cancer cells. Autophagy 10: 1495-1508, 2014.

31. Komatsu M, Wang QJ, Holstein GR, Friedrich VL Jr, Iwata J, Kominami E, Chait BT, Tanaka K and Yue Z: Essential role for autophagy protein Atg7 in the maintenance of axonal homeostasis and the prevention of axonal degeneration. Proc Natl Acad Sci USA 104: 14489-14494, 2007.

32. Le CP, Nowell CJ, Kim-Fuchs C, Botteri E, Hiller JG, Ismail H, Pimentel MA, Chai MG, Karnezis T, Rotmensz N, et al: Chronic stress in mice remodels lymph vasculature to promote tumour cell dissemination. Nat Commun 7: 10634, 2016.

33. Krizanova O, Babula P and Pacak K: Stress, catecholaminergic system and cancer. Stress 19: 419-428, 2016.

34. Huang B, Chen H, Deng Y, Yi T, Wang Y and Jiang Y: Diagnosis, disease stage, and distress of Chinese cancer patients. Ann Transl Med 4: 73, 2016.

35. Reiche EM, Nunes SO and Morimoto HK: Stress, depression, the immune system, and cancer. Lancet Oncol 5: 617-625, 2004.

36. Xie CM, Chan WY, Yu S, Zhao J and Cheng CH: Bufalin induces autophagy-mediated cell death in human colon cancer cells through reactive oxygen species generation and JNK activation. Free Radic Biol Med 51: 1365-1375, 2011.

37. Hardie DG: AMP-activated protein kinase: An energy sensor that regulates all aspects of cell function. Genes Dev 25: 1895-1908, 2011.

38. Li X, Yuan Z, Liu R, Hassan HM, Yang H, Sun R, Zhang L and Jiang Z: UDCA and CDCA alleviate $17 \alpha$-ethinylestradiol-induced cholestasis through PKA-AMPK pathways in rats. Toxicol Appl Pharmacol 311: 12-25, 2016.

This work is licensed under a Creative Commons Attribution-NonCommercial-NoDerivatives 4.0 International (CC BY-NC-ND 4.0) License. 